\title{
Effect of Fabric Pattern and Color on Impression Evaluation of Textile Images Rendered by a Textile Simulator
}

\author{
KyoungOk Kim ${ }^{\text {a }}$, Takanari Koyama ${ }^{\mathrm{b}}$, Yuu Takamizawa ${ }^{\mathrm{c}}$ \\ Chinami Fujii ${ }^{\mathrm{c}}$, Masayuki Takatera ${ }^{\mathrm{a}, *}$ \\ ${ }^{a}$ Division of Kansei and Fashion Engineering, Institute for Fiber Engineering (IFES), Interdisciplinary \\ Cluster for Cutting Edge Research (ICCER), Shinshu University, Ueda, Nagano, 386-8567, Japan \\ ${ }^{\mathrm{b}}$ Faculty of Textile Science and Technology, Shinshu University, Ueda, Nagano, 386-8567, Japan \\ ${ }^{\mathrm{c}}$ Graduate School of Science and Technology, Shinshu University, Ueda, Nagano, 386-8567, Japan
}

\begin{abstract}
The expression ability of a commercial textile simulator was investigated by simulating textile images taking into account the effect of yarn count, weave density, yarn types and weave. The performance of the simulator was investigated by evaluating and comparing impressions of real textiles and simulated images. The effect of pattern and textile color on the simulation performance was also studied. It was found that simulated images of textiles with more complicated and multicolored patterns were perceived to be more alike to the real textiles. Simulated images with large patterns were also evaluated and found to compare favorably to real textiles. Color was observed to have no effect on the impression of simulated textile images. Therefore, textile simulation in the textile trade is more effective on complex variations of textile patterns and colors.
\end{abstract}

Keywords: Performance; Textile Image; Textile Simulation; Color and Pattern

\section{Introduction}

Textile is made up of various yarns with various structures. Those affect the visual impression and the handle. The increased globalization of industry makes growing of internationalization in textile trading. In the international textile trade, many manufacturers participate in international exhibitions to present their products and locate new customers. Even in the domestic market, exhibitions are an important method of communicating with customers. However, time and monetary costs are high for exhibitors who wish to participate in international exhibitions, especially with regard to samples. Customers can request samples of differing colors, yarn type, weave and so on, and request that the new samples be posted to them. Making and sending

\footnotetext{
${ }^{*}$ Corresponding author.

Email address: takatera@shinshu-u.ac.jp (Masayuki Takatera).
} 
these samples takes time and costs money, particularly in the case of trading between countries. Furthermore, all this effort does not always result in an order, so the production of additional samples increases the risk quotient. For smaller textile companies, these kinds of sample-making processes are difficult, even though they produce attractive high value textiles. To solve those problems, businesses have introduced textile simulation in addition to textile design. In many cases it is possible to produce simulated textile samples, by textile simulator, which can convey the same visual image and touch characteristics as a real textile. In addition, this methodology, when done correctly, can reduce costs and save production time.

Many researchers have investigated the geometrical $[1,2]$ and physical properties of textiles [3-10] to simulate textiles effectively. Moreover, garment simulation is a popular field of investigation for many research groups [11-15]. The relevant technology has already been put to practical use in the simulation of textiles. Because of the necessity of simulators in the textile industry, several commercial simulators have been developed [16-21]. Using those simulators, three-dimensional impressions of warp and weft knitting fabrics can be expressed. In addition, it is also possible to express the derivative and multiple-weave woven fabrics. However, there are still some limitations in yarn and weave structure modeling [22]. The performance of textile simulators is not set for any particular selection of fabric, but this expression ability is an important part of simulator use.

In this study, we investigated the performance of a current commercial simulator. We investigated the expression ability of simulator taking into account the effect of yarn count, weave density, yarn types and weave on the fabric simulation. Then, we evaluated the impression of real textiles and of simulated images by a sensory test. Comparing these results, we investigated the performance of the textile Computer Aided Design (CAD) system. We also investigated the effect of pattern and textile color on the performance of simulated images by evaluating the agreement of the simulated images with the real textiles.

\section{Expression Ability of a Commercial Textile Simulator}

Before the sensory test, we investigated the expression ability of simulator taking into account the effect of yarn count, weave density, yarn types and weave on the fabric simulation using a textile CAD system (DesignScope victor Dobby, EAT GmbH, Germany [3]). This system is a yarn-based textile simulator, on which we could choose the 3D shape, color and texture of each yarn. We simulated various fabrics by controlling factors such as yarn count, weave density, yarn types and weave. With the images, we investigated the limitation of the simulation.

\subsection{Effect of Weave Density}

We simulated fabric images by controlling the weave density. Cotton yarn of 11.7 tex $\left(50 \mathrm{Ne}_{\mathrm{C}}\right)$ in the system was used for warp and weft yarns to simulate fabrics. The warp and weft densities were controlled as increasing $10 / \mathrm{cm}$ in warp and weft directions. Table 1 shows the simulated images. Fabric images of lower density expressed a gap between the yarn. In addition, fabric images of higher density with more than $40 / \mathrm{cm}$ both vertical and horizontal showed overlaped yarns and showed a moiré effect. It was understood that they were forcibly represented by overlapping the threads to each other. This exactly shows the weaving limit by the geometrical model [26]. 
Table 1: Simulated fabric images with different weave density for constant yarn count

Density of
weft yarn

\subsection{Effect of Yarn Count}

Fabrics were simulated by changing the yarn count. Cotton yarn in the system was used to simulate fabric images for constant warp and weft weave densities of $30 / \mathrm{cm}$. Table 2 shows the simulated fabric images with different yarn count in warp and weft directions. In the system, it was shown that the thickness of the yarn is accidentally changed by changing the density of the yarn, rather than a gap is changed. Thus, change of yarn thickness were unable to be expressed due to the thickness changes in conjunction with the density of the yarn. This also shows the weaving limit. 
Table 2: Simulated fabric images with different yarn count for constant weave density

\begin{tabular}{|c|c|c|c|c|c|}
\hline \multirow{2}{*}{$\begin{array}{c}\text { Weft yarn } \\
\text { count }\end{array}$} & \multicolumn{5}{|c|}{ Warp yarn count } \\
\hline & 6.56 tex $\left(90 \mathrm{Ne}_{\mathrm{C}}\right)$ & 8.44 tex $\left(70 \mathrm{Ne}_{\mathrm{C}}\right)$ & 11.8 tex $\left(50 \mathrm{Ne}_{\mathrm{C}}\right)$ & 19.7 tex $\left(30 \mathrm{Ne}_{\mathrm{C}}\right)$ & 59.1 tex $\left(10 \mathrm{Ne}_{\mathrm{C}}\right)$ \\
\hline $\begin{array}{l}6.56 \text { tex } \\
\left(90 \mathrm{Ne}_{\mathrm{C}}\right)\end{array}$ & 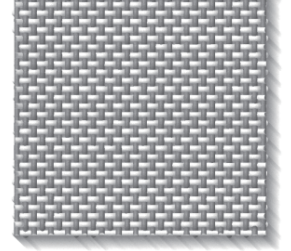 & 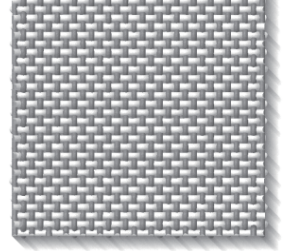 & 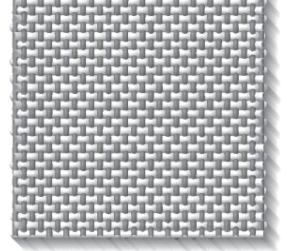 & 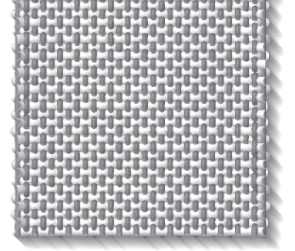 & \\
\hline $\begin{array}{l}8.44 \text { tex } \\
\left(70 \mathrm{Ne}_{\mathrm{C}}\right)\end{array}$ & 礛 & 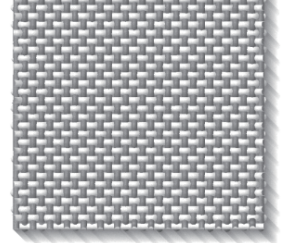 & 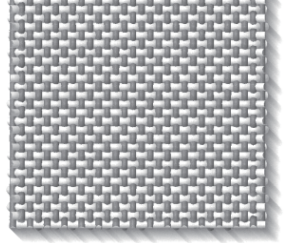 & 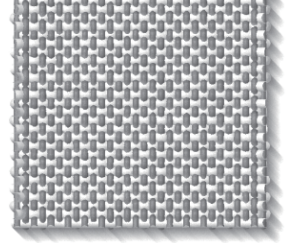 & ate \\
\hline $\begin{array}{l}11.8 \text { tex } \\
\left(50 \mathrm{Ne}_{\mathrm{C}}\right)\end{array}$ & & 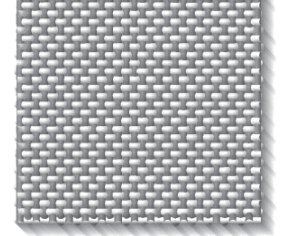 & 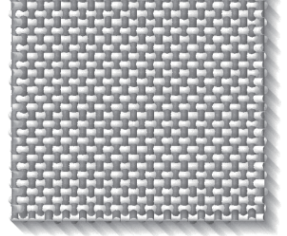 & 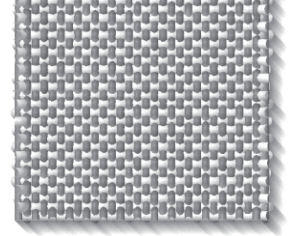 & 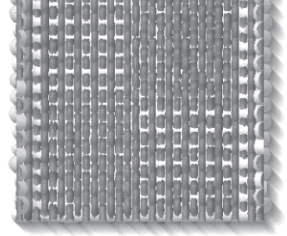 \\
\hline $\begin{array}{c}19.7 \text { tex } \\
\left(30 \mathrm{Ne}_{\mathrm{C}}\right)\end{array}$ & & 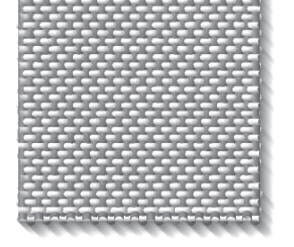 & 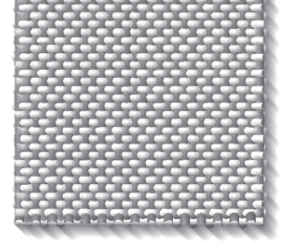 & 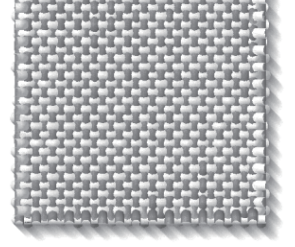 & $\begin{array}{r}4 \\
8 \\
8 \\
8 \\
8\end{array}$ \\
\hline $\begin{array}{c}59.1 \text { tex } \\
(10 \text { count })\end{array}$ & 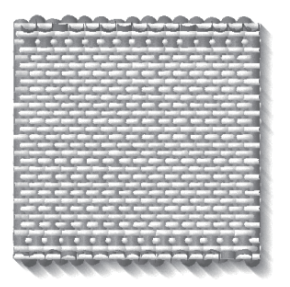 & 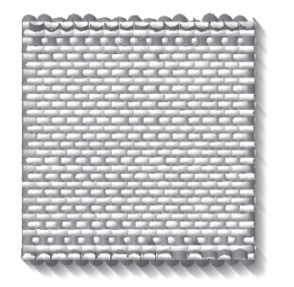 & 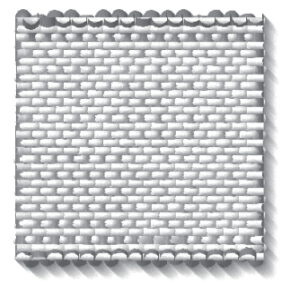 & 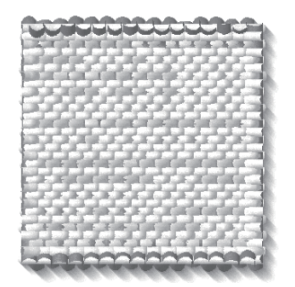 & S \\
\hline
\end{tabular}

\subsection{Effect of Weave Structure}

Fabrics were simulated by controlling weave structure and yarn material. We set 5 types of weave structures, such as plain, 3/1 twill, 5 harness satin, mock leno and honey-comb weave and 3 types of yarn materials for constant warp and weft weave densities of $30 / \mathrm{cm}$ with yarn counts of 11.7 tex to confirm the effect of structure on simulated fabric image.

We simulated fabric images with cotton yarn and 5 types of weave structures. The images are shown in Table 3. To simulate images, we used EAT-standard yarns which were preset in the system. Table 3 showed the simulated images with different weave structures and yarn types. All of the surfaces are smooth, and the texture also can be felt in its own way. For fabric images of 
mock leno and honey comb weaves, they showed change of the yarn interval by weave. However in-plane deflection of yarns and the three-dimensional surface structures were not shown enough in comparison with real fabrics. It was found that the system can express a certain level of the three-dimensional deformation of yarns in special weaves.

The simulated images with woolen yarn and 5 types of weave structures are also shown in Table 3. We used preset EAT-spun-swell yarn which has fuzz on the surface. Similar to images with cotton yarn, the texture of woolen yarn were represented in its own way. Although the recognition of boundary of the yarn becomes more difficult than the fabric images of cotton yarn due to its fluff, the fine patterns due to weaves were clearly shown. For the three-dimensional effect, although the yarn deflection is observed compared to cotton on the image of honey-comb and mock leno weaves, three-dimensional representation still remained unsatisfactory. This simulator does not consider the difference in mechanical properties of the yarn. Therefore, the visual effect of the yarn deflection depends on the surface properties of the yarn model. It was also found that the unevenness of the woollen yarn was not expressed sufficiently.

The simulated images with silk yarn and 5 types of weave structures are also shown in Table 3. We used preset EAT-silky yarn which has luster. In terms of texture, luster is firmly expressed which was different with other yarns. Boundary of the yarn was clearly expressed than the others. Three-dimensional appearance of the thread is also clearer than the others. However, no great difference was shown with other yarns. The three dimensional effects by structures were still insufficiently represented.

\section{Experiments}

\subsection{Impression Evaluation of Simulated Images}

To investigate the performance of a commercial textile CAD system, we compared the impression of real textiles and of simulated images. We prepared 5 kinds of textile samples and their associated simulated images using a textile CAD system (DesignScope victor Dobby, EAT GmbH, Germany [3]). The fabrics was inspected and the most appropriate model, color and texture for the simulation of each yarn was chosen. The weave and weave-densities were set to the same as the real textile samples.

Five textile samples that each had different surface patterns were selected. The specifications of these textile samples are shown in Table 4. Fig. 1 shows the real textile samples and Fig. 2 shows the simulated images.

Using the real textile samples and the simulated images, we asked a group of subjects (15 university students; 1 male, 14 females) to carry out a sensory evaluation test using the following three steps:

(1) Subjects evaluated the fabrics by watching the simulated image on a display.

(2) Subjects evaluated the fabrics by looking at the real planar fabric, but without touching it.

(3) Subjects evaluated the fabrics by looking at and touching the real fabric.

They rated the evaluation items of each image and fabric pair on a scale of 1 to 5 , as shown in Table 5 .

During the test, the subjects first sat in front of a computer monitor (FlexScanSX2762W, EIZO 
Table 3: Simulated fabric images with different weave and yarn types

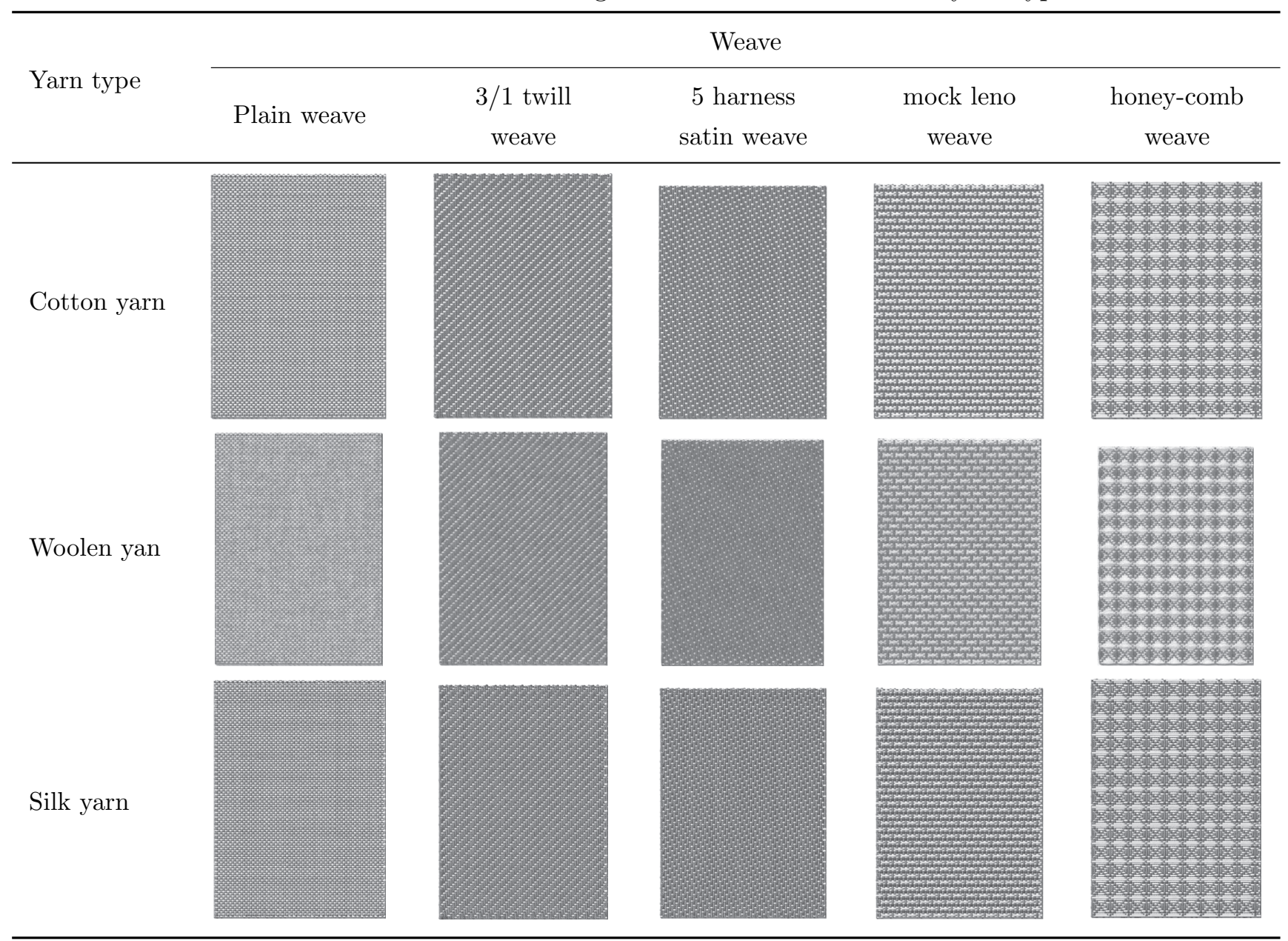

Corporation, Ishikawa Japan), watched the simulated images presented on the computer screen, and rated each image. Then, the real textile sample was presented, which they also scored by observing it. Finally, they scored the real textile sample by looking at and touching it.

Analysis was carried out of variance. We also performed a significance test between samples, which showed differences in the average by multiple comparisons.

In addition, we asked the subjects the following question "You ordered a product made from

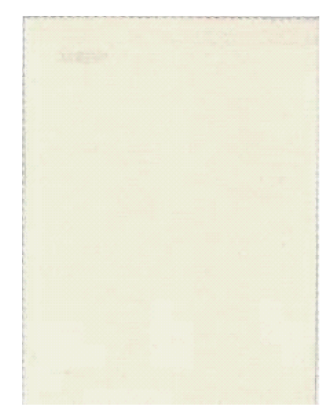

(a) $\mathrm{A}$

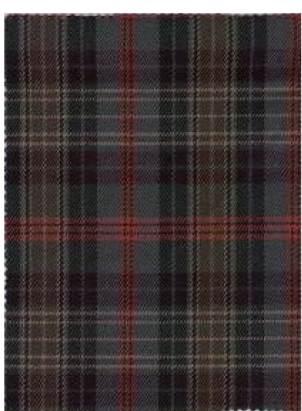

(b) B

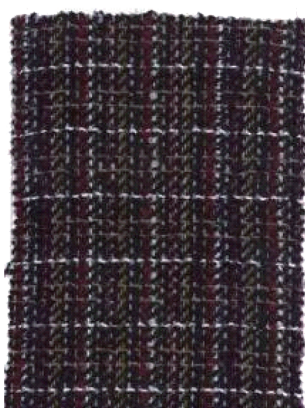

(c) $\mathrm{C}$

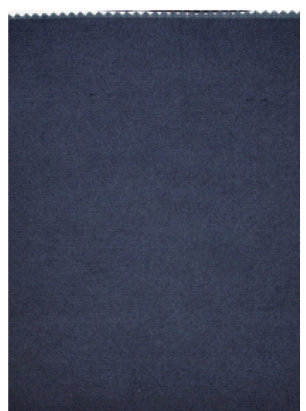

(d) D

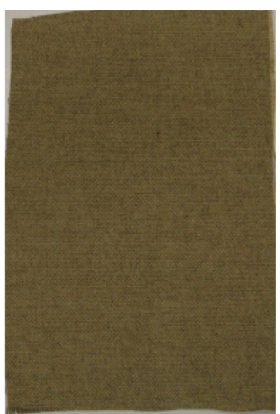

(e) E

Fig. 1: Scanned images of real fabrics 


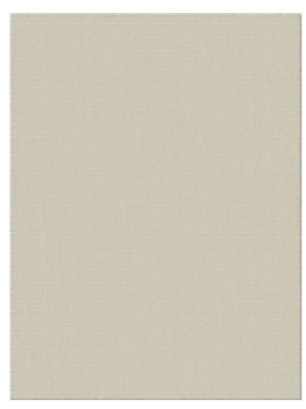

(a) A

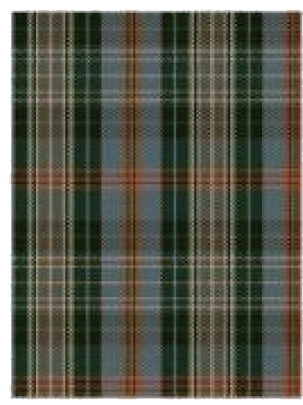

(b) B

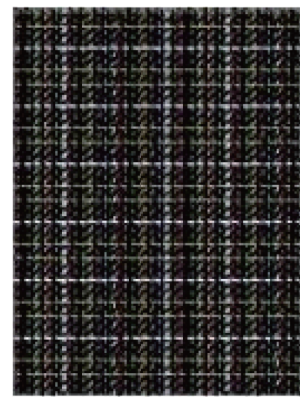

(c) $\mathrm{C}$

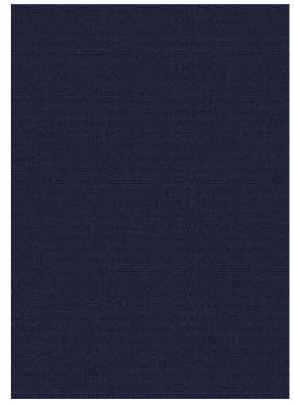

(d) D

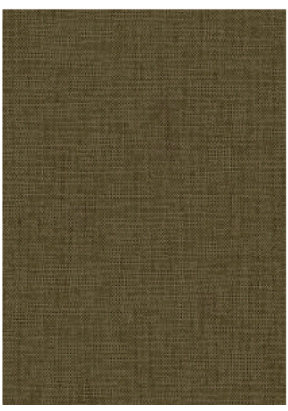

(e) E

Fig. 2: Simulated images of fabrics

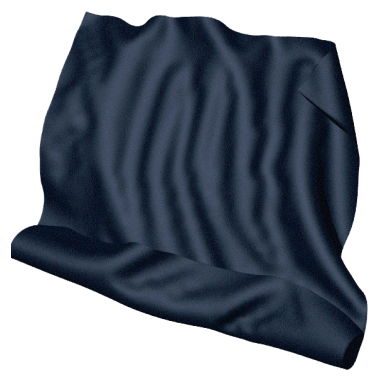

Fig. 3: Three-dimensional shape of sample D, rendered by textile CAD

Table 4: Sample characteristics

\begin{tabular}{cccccc}
\hline $\begin{array}{c}\text { Sample } \\
\text { name }\end{array}$ & Structure & Pattern & Material & $\begin{array}{c}\text { Yarn density (tex) } \\
\text { warp } \times \text { weft }\end{array}$ & $\begin{array}{c}\text { Weave density }(/ \mathrm{cm}) \\
\text { warp } \times \text { weft }\end{array}$ \\
\hline A & $1 / 2$ twill & plain & wool 100\% & $32 \times 32$ & $35 \times 35$ \\
B & $3 / 3$ twill & check & wool $50 \%$, polyester $50 \%$ & $45 \times 45$ & $35 \times 24$ \\
C & $2 / 2$ twill & check & wool $85 \%$, nylon $11 \%$, & fancy yarn & $7 \times 8$ \\
D & $1 / 4$ twill & plain & silk $100 \%$ & $48 \times 2$ & $30 \times 60$ \\
E & plain & melange & wool $52 \%$, polyester $100 \%$ & $78 \times 78$ & $13 \times 13$ \\
\hline
\end{tabular}

Table 5: Evaluation items for impression test

\begin{tabular}{c} 
Hard-soft \\
Heavy- light \\
Thick-thin \\
Warm-cold \\
Stiff-flexible \\
Rough-smooth \\
\hline
\end{tabular}

this textile sample after seeing simulated images in the online shopping. When you received the real product, how does it feel? Would you accept it?". For this question, we prepared an additional image of sample D, which was rendered three-dimensionally as shown in Fig. 3. 


\subsection{Effect of Color and Pattern on Simulated Images}

In this experiment, we investigated the effect of color and pattern on the performance of textile simulation. We prepared 12 real textile samples; four colors (yellow, blue, black and red) of three different kinds of pattern (hound's tooth, hairline and zigzag) [23]. The textile patterns were made using different combinations of yarn color, the specifications of which are shown in Table 6. The real textile samples for each pattern, in black and white, are shown in Fig. 4.

Table 6: Textile sample characteristics

\begin{tabular}{|c|c|c|c|c|c|c|}
\hline Sample & Structure & Pattern & Color & Material & $\begin{array}{c}\text { Yarn density }(\text { Tex) } \\
\text { warp } \times \text { weft }\end{array}$ & $\begin{array}{c}\text { Density }(/ \mathrm{cm}) \\
\text { warp } \times \text { weft }\end{array}$ \\
\hline $\mathrm{F}$ & Twill & Hound's tooth & Black and white & \multirow{12}{*}{$\begin{array}{l}48 \% \text { wool, } \\
38 \% \text { cotton, } \\
\text { nylon } 14 \%\end{array}$} & \multirow[t]{12}{*}{45} & \multirow[t]{12}{*}{$20 \times 20$} \\
\hline G & & & Yellow and white & & & \\
\hline $\mathrm{H}$ & & & Blue and white & & & \\
\hline I & & & Red and white & & & \\
\hline $\mathrm{J}$ & & \multirow[t]{4}{*}{ Hairline } & Black and white & & & \\
\hline K & & & Yellow and white & & & \\
\hline $\mathrm{L}$ & & & Blue and white & & & \\
\hline M & & & Red and white & & & \\
\hline $\mathrm{N}$ & & \multirow[t]{4}{*}{ Zigzag } & Black and white & & & \\
\hline $\mathrm{O}$ & & & Yellow and white & & & \\
\hline $\mathrm{P}$ & & & Blue and white & & & \\
\hline $\mathrm{Q}$ & & & Red and white & & & \\
\hline
\end{tabular}

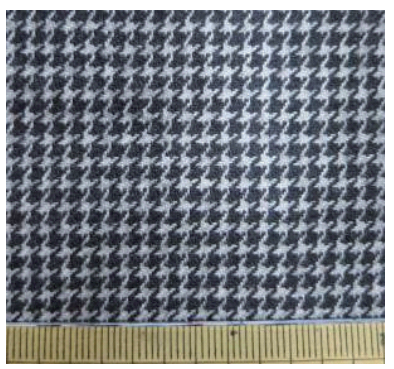

(a) Hound's tooth

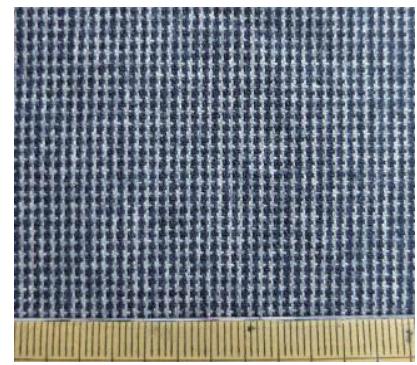

(b) Hairline

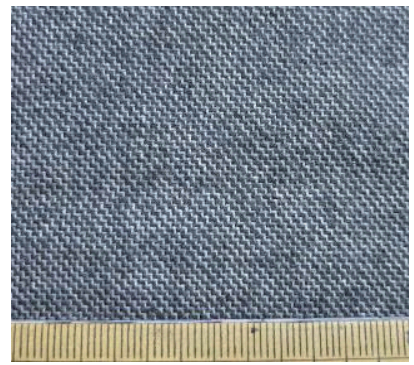

(c) Zigzag

Fig. 4: Real textile samples in black and white

We then simulated the textile samples. The black and white simulated images are shown in Fig. 5. In the simulation, the textile color was matched visually on the monitor (FlexScanSX2762W, EIZO). To investigate the effect of color, we simulated 60 fabrics. These comprised white yarn with 20 colors of yarns for each of the three patterns, as shown in Table 7 . Of these 20 colors, every 5 colors belonged in each 4 hue regions (yellow, blue, black and red system). We used those simulated images and the real textiles for sensory tests.

Experiments were carried out in two ways. First, subjects evaluated the evaluation items of the simulated images and scored them on a scale of 1 to 7 , as shown in Table 8. The evaluation items were selected based on the results of experiment 2.1 and on the requirements for fabric selection [24]. The display size of the image on the monitor was adjusted to the size of real textile sample. 


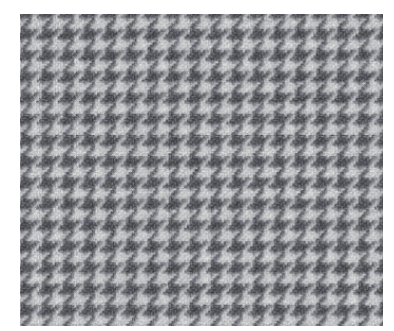

(a) Hound's tooth

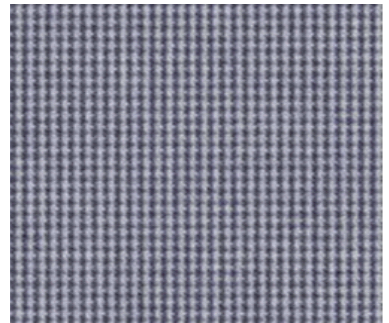

(b) Hairline

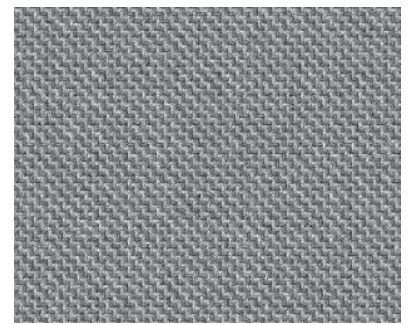

(c) Zigzag

Fig. 5: Simulated images in black and white

Table 7: Specification of simulated images

\begin{tabular}{|c|c|c|c|c|}
\hline \multirow{2}{*}{ Pattern } & \multicolumn{4}{|c|}{ Hue region } \\
\hline & yellow & blue & black & red \\
\hline \multicolumn{5}{|c|}{ Hound's tooth } \\
\hline \multicolumn{5}{|l|}{ Hairline } \\
\hline Zigzag & & & & \\
\hline
\end{tabular}

The subjects evaluated the yellow hue region simulated images for three patterns, for a total of 15 sheets, as described in Table 7 . In a similar manner, the simulated images of the black, blue, and red hue regions were evaluated.

Second, subjects evaluated the real textile samples and scored them the same evaluation items. The four color textiles (order: yellow, blue, black and red) were evaluated first in the hound's tooth pattern then in the hairline pattern and finally the zigzag pattern.

Results of the simulated images and the real textile samples were compared and the degree of agreement of the evaluation items was investigated. We analyzed the variance of the evaluation results, and carried out a significance test between samples, which showed the difference in the averages, by multiple comparisons.

The same final question of experiment 2.1 was asked of the subjects, using three textiles of each pattern. In addition, the subjects compared the difference of the materials and patterns between the simulation images and the real textiles, on a scale of 1 to 7 . The subjects for this experiment were 15 students ( 7 males, 8 females), and the average age was 22.9 years (standard deviation 1.9). Experiments were all conducted under fluorescent lighting, in a standard room.

Table 8: Items for impression test

\begin{tabular}{c} 
Hard-soft \\
Thick-thin \\
Wet-dry \\
Rough-smooth \\
\hline
\end{tabular}




\section{Results and Discussion}

\subsection{Evaluation of Impression in Simulated Images}

Fig. 6 shows the comparison of results, for the three evaluation methods, for sample A. Significant differences were seen between the simulated images and the real textile samples in the test where subjects could only look at the samples. All evaluation items showed differences except for the 'warm-cold'. For the method in which the subjects could touch the samples, significant differences were seen for all evaluation items between the simulated images and the real textile samples. In contrast, no significant differences were seen for either watching or touching the real textile samples, with the exception of the 'warm-cold' item. Thus, we found that it was difficult to provide significant information about the simulation of sample A.

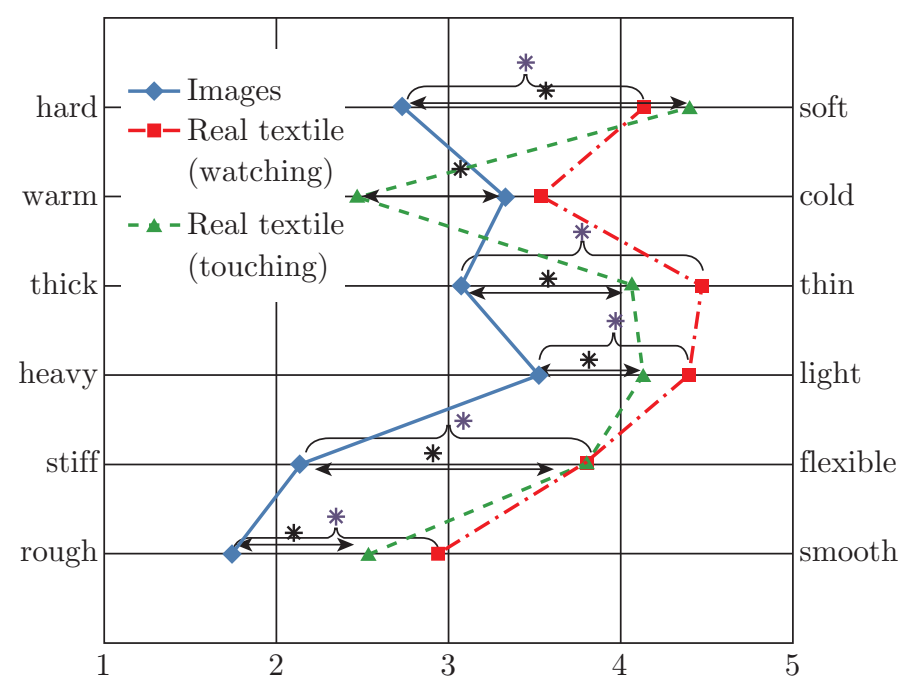

Fig. 6: Comparison of the three evaluation methods for sample A

Fig. 7 shows the comparison of results for the three evaluation methods for sample B. For sample $\mathrm{B}$, there were no significant differences between the simulated images and the visual evaluation of real textiles, excepting the 'hard-soft' item. However, significant differences were observed between simulated images and real textiles when they were evaluated by touching, except for the 'warm-cold' item. Thus we found that even though the simulation was visually close to the real sample, the tactile impression was quite different for sample B.

Sasaki and Shimizu [25] investigated the effect of mechanical fabric properties on visual tactile impression. They found that people form their first impression of cloth visually, before touching the fabric. Tactile impression is accumulated after touching the textile. When a subject looked at sample B, it may be that they imagined how the fabric would feel, based on their previous experience. However, their imagination would differ from the real tactile impression.

Fig. 8 shows the comparison of results for the three evaluation methods for sample C. There were no significant differences in the looking-only method between the simulated images and the real textiles, except for the item of 'warm-cold'. No significant differences were seen in the touching method, except for the evaluation items of 'hard-soft' and 'heavy-light'. Significant differences were seen, however, between the evaluation of the real textile samples by watching or by touching, especially in the evaluation items of 'warm-cold' and 'thick-thin'. In comparison with 


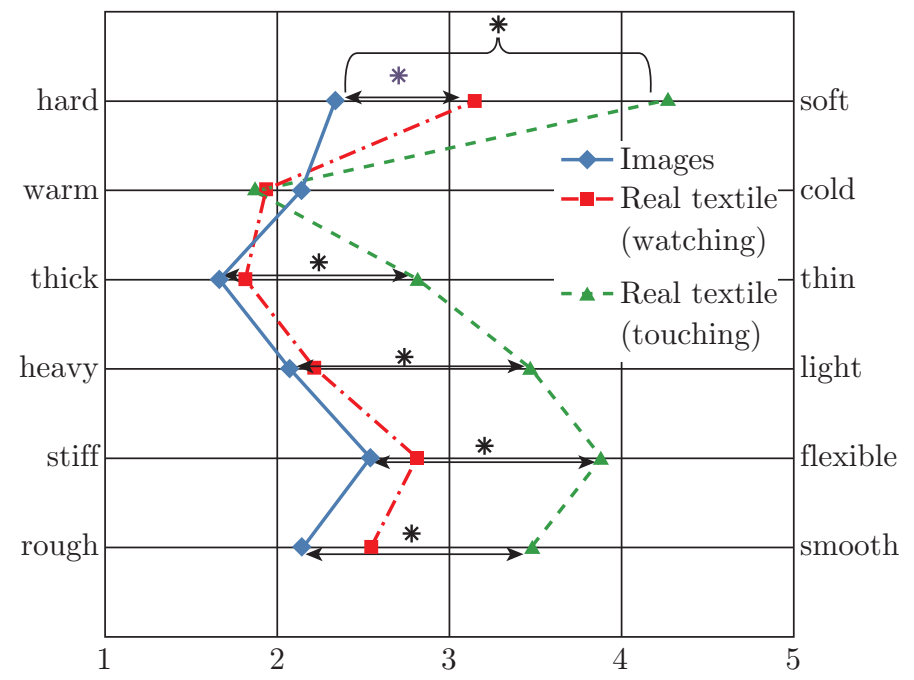

Fig. 7: Comparison of the three evaluation methods for sample B

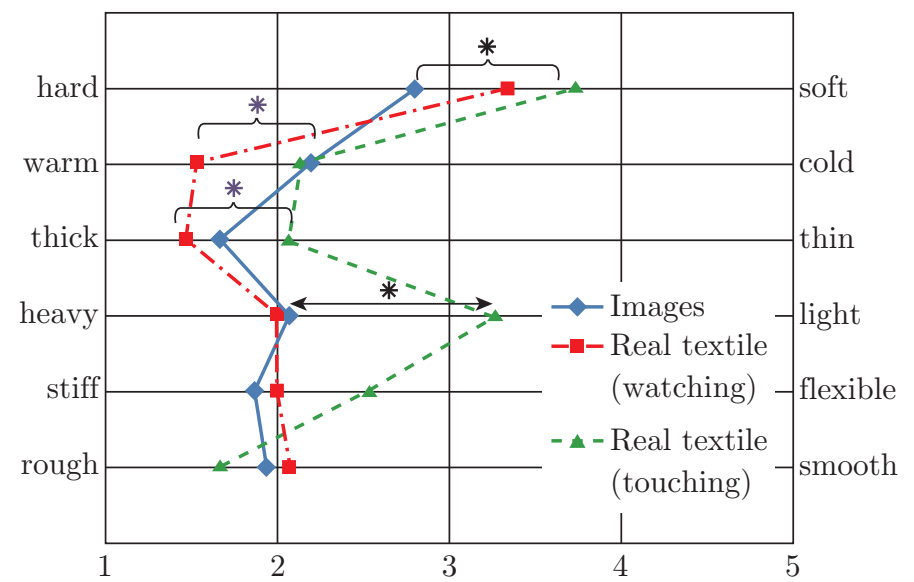

Fig. 8: Comparison of the three evaluation methods for sample $\mathrm{C}$

the results of other fabrics, the numbers of evaluation items that showed significant differences were relatively small. As a result, we found that the visual impressions of the real fabric and the simulated image were similar to each other. The associated-feel was relatively close to the real-feel for this sample.

Fig. 9 shows the comparison of results of three evaluation methods for sample D. There were significant differences between simulated images and the real textile samples for the watching method, in the 'thick-thin', 'heavy-light' and 'stiff-flexible'. The touching method showed significant differences between the simulated images and the real textiles in the 'hard-soft', 'warm-cold' and 'thick-thin' evaluation items. The comparison of the real textiles either by observing or by touching also produced significant differences in these evaluation items. As there are many significant differences, it was assumed that the simulated images were indicative of the real textile sample.

Fig. 10 shows the comparison of results for the three evaluation methods for sample E. Except for the 'rough-smooth' evaluation items, there were significant differences between the results of the simulated images, real samples (watching) and real samples (touching), respectively. As a result, it was found that the simulated image was not similar to the real textile sample. There 


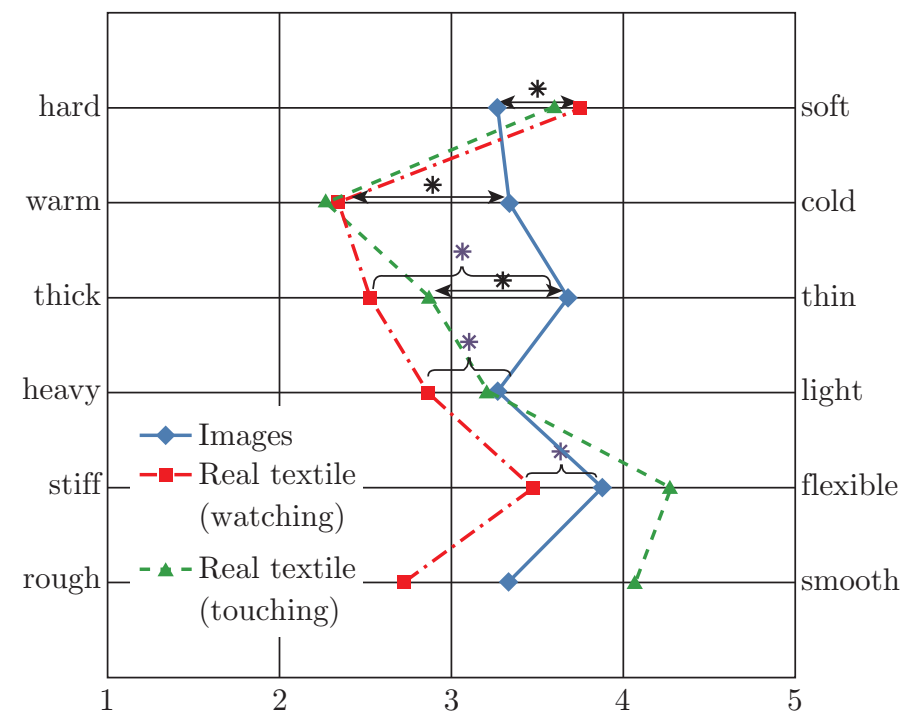

Fig. 9: Comparison of the three evaluation methods for sample D

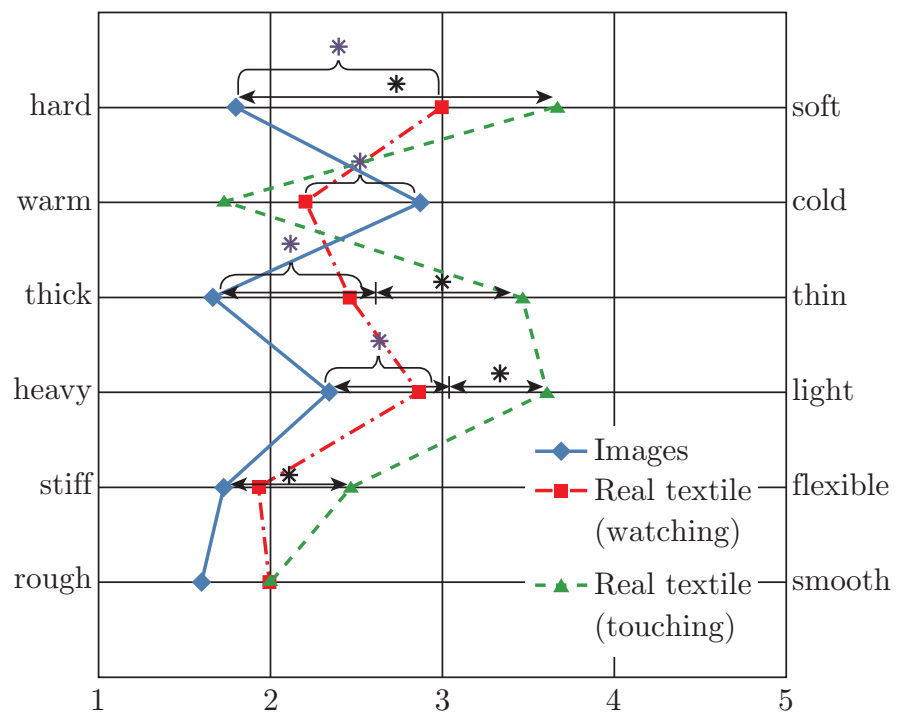

Fig. 10: Comparison of the three evaluation methods for sample E

were even differences between either watching or touching the real textile samples. Many subjects mentioned that they had not experienced textiles similar to sample D. Thus, they had no preconceived ideas about how it felt.

Subjects could not imagine how samples D and E would feel based on only the visual images. These fabrics had a plain surface and pattern. Thus, it was difficult to estimate the fabric structure, making it hard to imagine how they would feel. As a result, the performance of simulated images for Sample C was the highest, followed by that for samples B, A, D, and E. Consequently, it was found that if the real fabric was well-estimated from a surface image, the feel of the sample was also able to be guessed at. It was easy to estimate the touch of samples B and $\mathrm{C}$, but not the other fabrics.

The results of the additional the online shopping question are shown in Fig. 11. Sample B demonstrated lower performance, showing in Fig. 7, showed higher acceptability than one of 


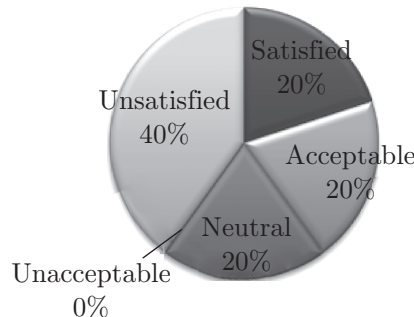

(a) $\mathrm{A}$

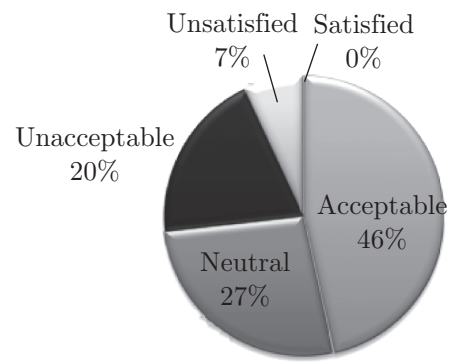

(d) D

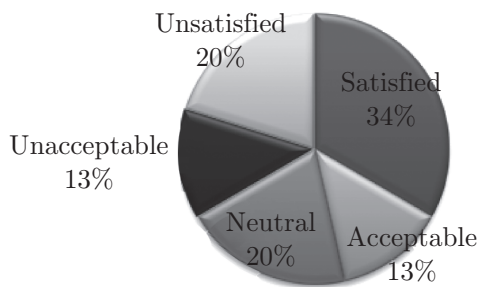

(b) B

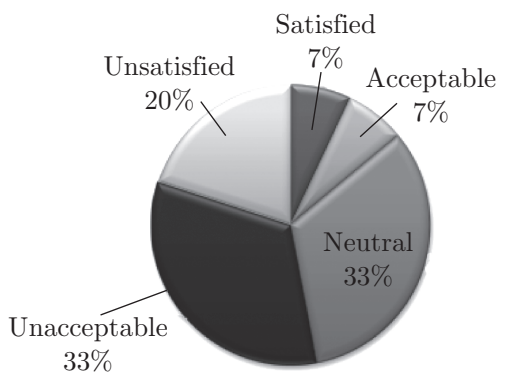

(e) $\mathrm{E}$

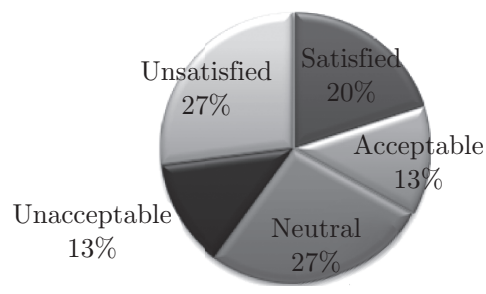

(c) $\mathrm{C}$

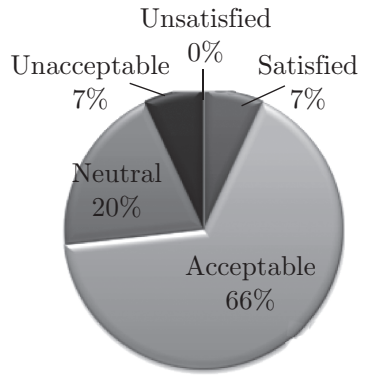

(f) Three-dimensional shape of sample D

Fig. 11: Results of addition online shopping satisfaction question

Sample C of higher performance, showing in Fig. 8. Subjects mentioned that there were differences between the impressions they formed about the product in their imagination, and the real product. They imagined the impression of textile assuming a product made by the textile. These differences in impression affected the satisfaction they felt. Furthermore, in the simulation of sample D, the degree of satisfaction of the planar simulated image did not show a high value (46\%), as presented in Fig. 11 (d). In contrast, the degree of satisfaction of the three-dimensionally simulated image showed a higher value $(73 \%$, Fig. 11 (f)). This was because of the luster characteristic of silk satin, as in sample D. This has been simulated very well in the three-dimensionally simulated image.

\subsection{Effect of Color and Pattern on Simulation}

Figs. 12 and 13 show the mean scores of the evaluation items for the simulated images and the

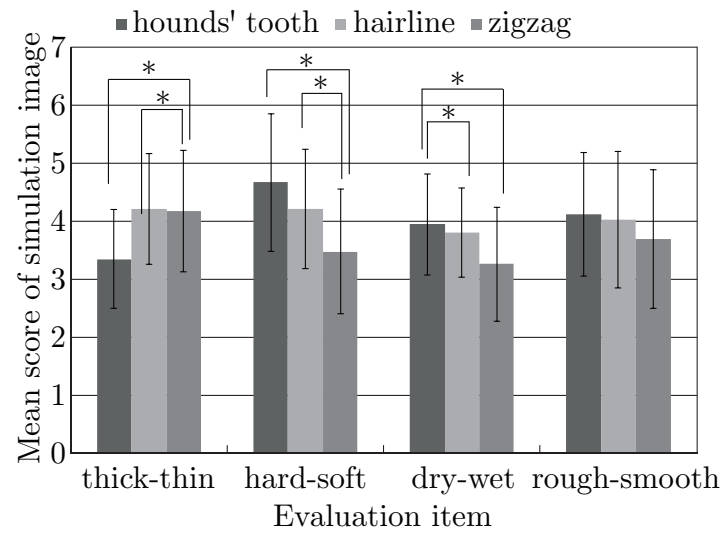

Fig. 12: Mean scores of simulated image evaluation, with respect to patterns 


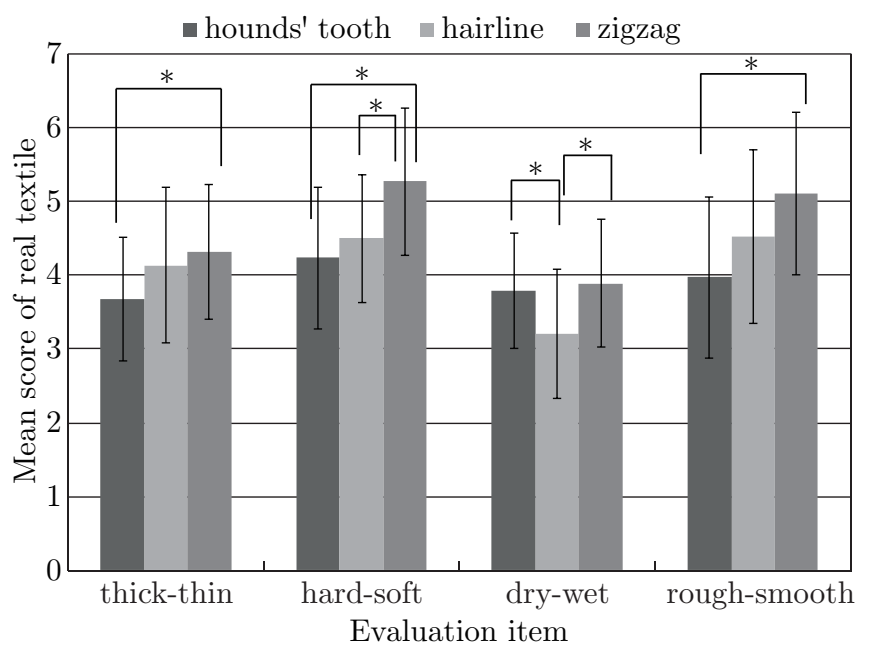

Fig. 13: Mean scores of real textile evaluation, with respect to patterns

real textiles, depending on the pattern types, respectively. There were significant differences between the average pattern evaluations for both simulated images and real textiles, except for the 'rough-smooth' item in the simulated images. Therefore, there are differences in the subjects' perceptions that are dependent on the patterns. The 'rough-smooth' item could be estimated from the surface image and could not be influenced by pattern size.

Figs. 14 and 15 show the mean scores of the evaluation items between the simulated images and the real textiles with regard to the hue region. There was no significant difference between hue regions in the simulated images and the real textiles. Thus, it was found that color did not influence the evaluation items.

Figs. 16 and 17 show the comparison of the mean scores of the simulated images and real textiles and their dependence on the pattern and hue region. Significance tests were carried out on the evaluation items for the same pattern and for the same hue region, respectively. In the case of pattern comparison, significant differences were found in the 'soft-hard' item between the simulated image and real fabric of hound's tooth pattern, and between the simulated image and the real fabric of zigzag pattern. In the 'wet-dry' item, significant differences were found

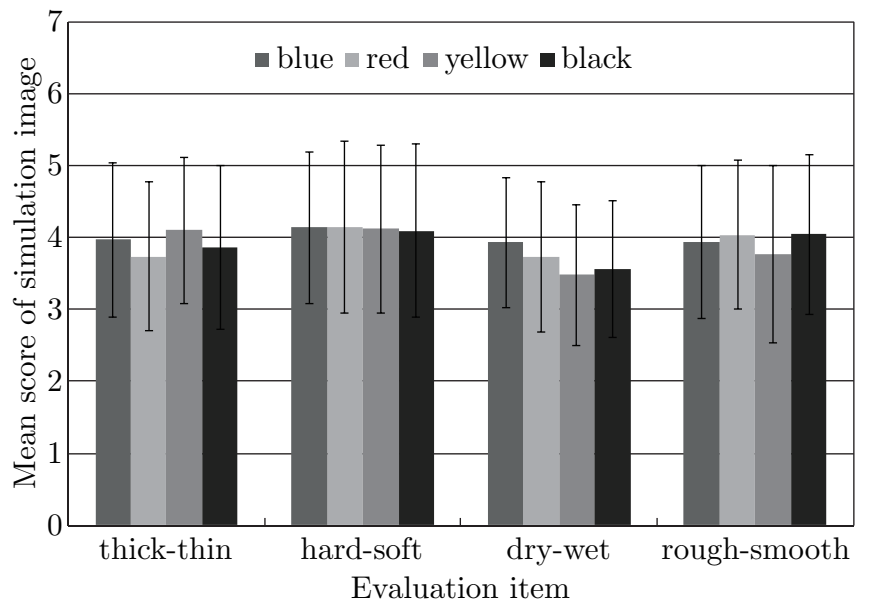

Fig. 14: Mean score of simulated image evaluation, with respect to hue region 


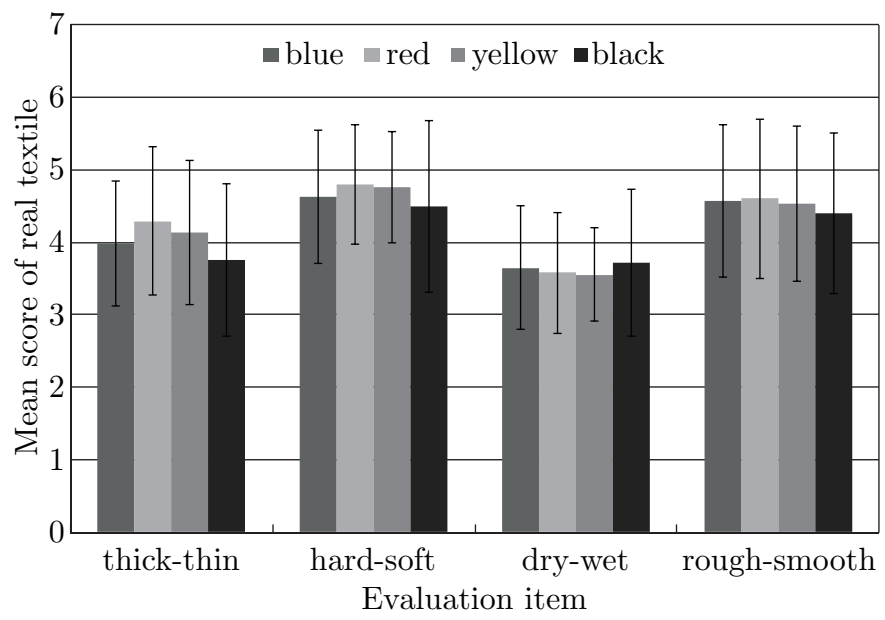

Fig. 15: Mean score of real textile evaluation, with respect to hue region

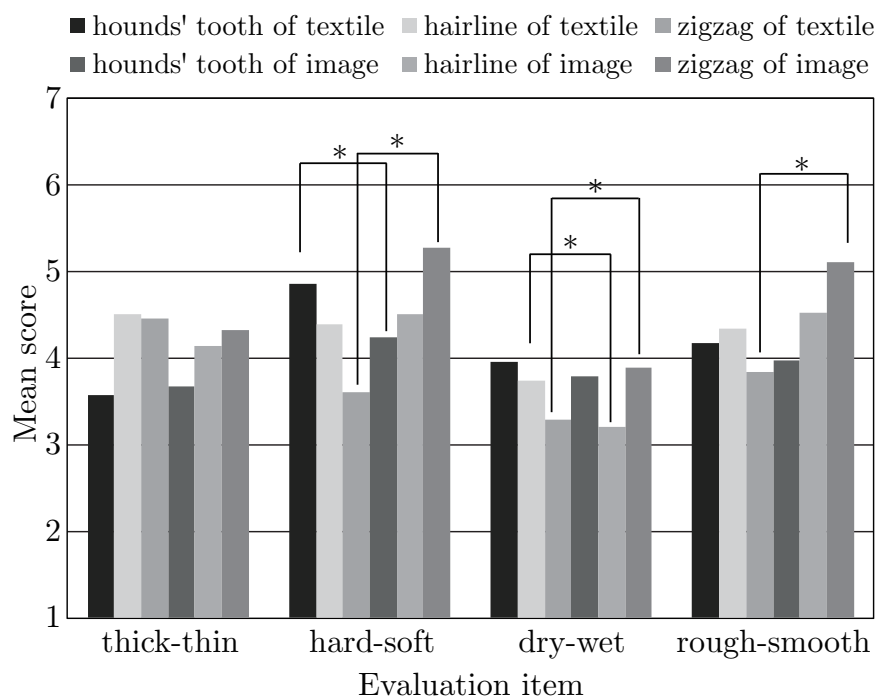

Fig. 16: Comparison of the mean score of simulated images and of real textiles, with respect to pattern

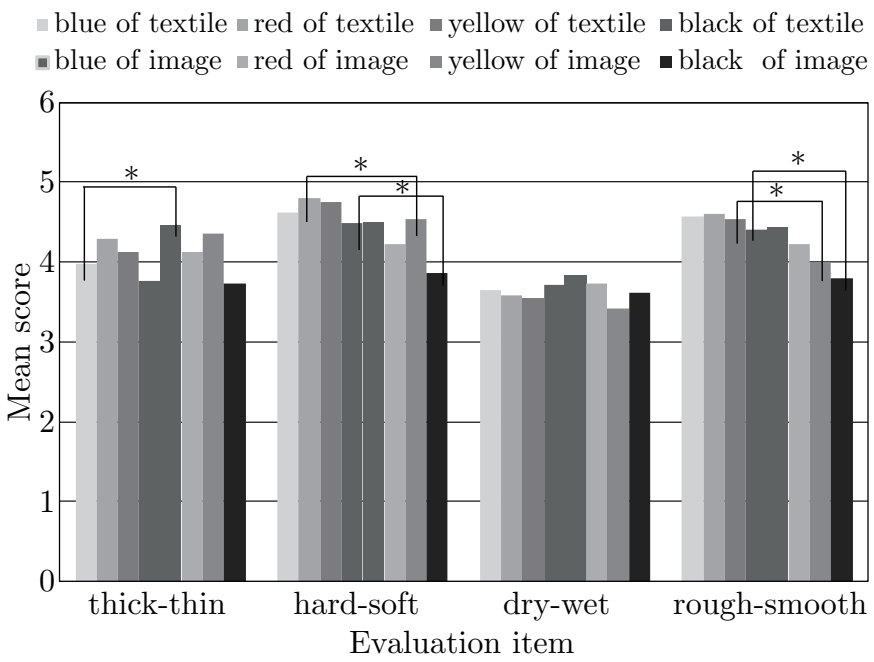

Fig. 17: Comparison of the mean score of simulated images and of real textile, with respect to hue region 
between the simulated image and real textile in both the zigzag and hairline patterns. In the 'rough-smooth' item, significant differences were shown between the simulated image and the real textile of zigzag pattern. In the comparison of color, differences were seen between the simulated images and the real fabric in the following evaluation items: blue hue region, 'thick-thin'; red and black hue region, 'hard-soft'; yellow and black hue region, 'rough-smooth'.

Fig. 18 shows the comparison of pattern and material difference between the simulated images and the real textile. Fig. 19 shows the degree of satisfaction for the simulated image for the online shopping purchase question. As order of hound's tooth, hairline and zigzag patterns, the material and the pattern were judged to be close to the real textile. The satisfaction score of the hound's tooth pattern was the best for the online shopping purchase, followed by the hairline and then the zigzag patterns. We therefore found that the simulated image of the large pattern textile was evaluated as similar to the real textile.

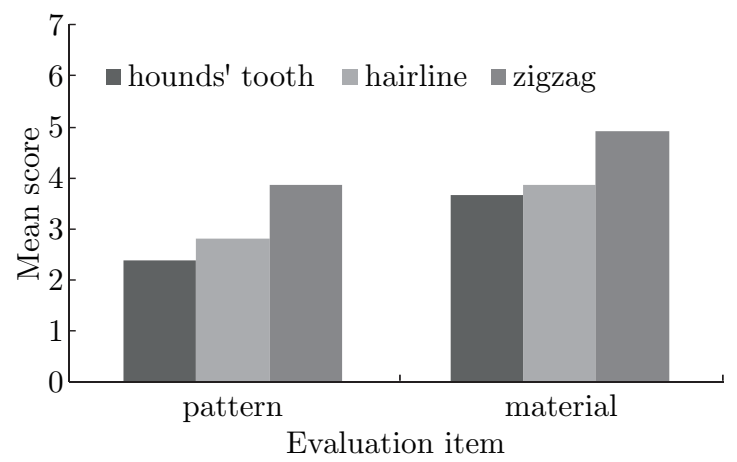

Fig. 18: Pattern and material difference of simulated images and real textile, with respect to pattern

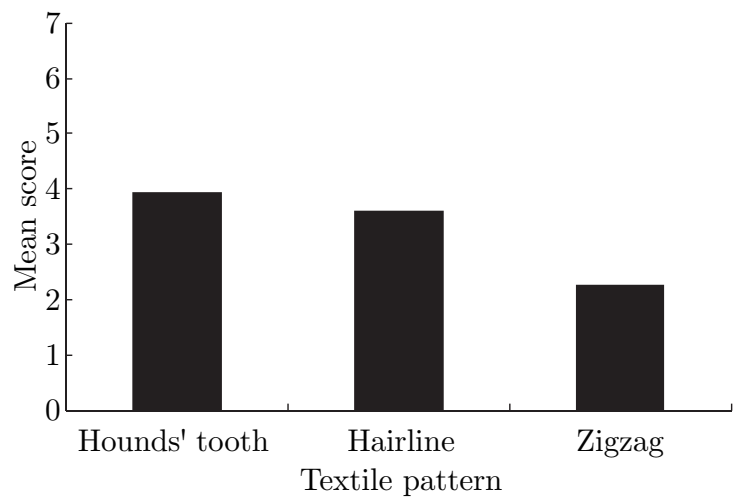

Fig. 19: Degree of satisfaction for simulated images, by online shopping purchase, with respect to pattern

\section{Conclusion}

We investigated the expression ability of a commercial textile simulator by simulating textile images taking into account various yarn count, weave density, yarn type and weave structure. The performance of a commercial textile simulator was also investigated by comparing simulated images and real textiles. It was found that simulated images of textiles with more colors and more complicated patterns were evaluated to be more like the real textile. For plainer surface textiles, it 
seems that more detailed information is necessary to ascertain the difference. Three-dimensional images will be effective in improving the textile performance, and improve the estimation of fabric properties. In other words, recognition has a limit even if the surface image looks real.

It was found that there is a possibility that the differences between the visual and tactile impression depends on the subjects' experience of textiles. Future study will be necessary to determine the effect of the subjects' experience of textiles on the impression of textile simulation.

With regard to the effect of pattern and color, it was found that impression of the simulated image was different depending upon the size of patterns. In particular, the simulated images with a large pattern were judged to be more similar to the real textiles with higher satisfaction. In the simulation of fabric with pattern, visual similarity is important Judgment of similarity of planar fabrics simulation is stricter than fabric with pattern. Therefore, in the textile trade, the simulator is expected to be more effective on the simulation of patterned textiles, as opposed to plain. This result is connected to the result of the check-patterned textiles in the first experiment. With regard to the evaluation items, color had no effect on the impression of simulated images or real textiles. Simulation will therefore be effective in showing the color variation of a real sample.

In addition, the degree of agreement of visual impression did not always correspond to the degree of satisfaction experienced by the test subjects. Further research about textile recognition will be necessary.

\section{Acknowledgement}

This work was supported by Japan Society for the Promotion of Science KAKENHI under Grant number 23240100.

\section{References}

[1] X. Xiao, T. Hua, L. Li, J. Wang, Geometrical modeling of honeycomb woven fabric architecture, Textile Research Journal, doi: 10.1177/0040517514548754, 2015

[2] S. V. Lomov, T. Mikolanda, M. Kosek, I. Verpoest, Model of internal geometry of textile fabrics: Data structure and virtual reality implementation, Journal of the Textile Institute, 98(1), 2007, $1-13$

[3] A. Briggs-Good, K. Townsend, Textile Design: Principles, Advances and Applications, Woodhead Publishing, 1st edition, 2011, 46-48

[4] iTex by NedGraphics (https://itunes.apple.com/jp/app/itex-by-nedgraphics/id522403867?mt=8)

[5] EAT GmbH, The DesignScope Company, (www.DesignScopeCompany.com)

[6] TexPro Design CAD System, YoungWoo CnI INC, (http://yuka-alpha.com)

[7] SHIMA SEIKI, SDS-ONE APEX3 (http://www.shimaseiki.com/)

[8] Digital Fashion Ltd, (http://www.digitalfashion.jp/)

[9] T. Liao, A. Sabit, A novel approach to three-dimensional modeling of interlaced fabric structures, Textile Research Journal, 68.11, 1998, 841-847

[10] O. Kouichi, I. Tatsuharu, Three-dimensional Computer Modeling of Woven Fabric Structures, Sen'i Kikai Gakkaishi, Journal of the Textile Machinery Society of Japan, Vol. 57, No. 8, 2004, T81-T88 (in Japanese) 
[11] S. Inui, H. Okabe, M. Takatera, M. Hashimoto, Y. Horiba, A fabric simulation based on a model constructed from a lower level, In Computational Engineering in Systems Applications, IMACS Multiconference, Vol. 1, 2006, 178-180

[12] S. V. Lomov, T. Mikolanda, M. Kosek, I. Verpoest, Model of internal geometry of textile fabrics: Data structure and virtual reality implementation, Journal of the Textile Institute, 98.1, 2007, $1-13$

[13] Z. Deng, L. Wang, Enhanced visualization of simulated woven fabrics, Fibers and Polymers, 11.3, 2010, 531-536

[14] S. Inui, Y. Mesuda, Y. Horiba, M. Kurihara, Fabric weave form simulation based on the characteristics of yarn, In Biometrics and Kansei Engineering (ICBAKE), IEEE, 2011, 49-52

[15] S. Inui, Y. Mesuda, Y. Ushiyama, M. Kurihara, Y. Horiba, Improvement of fabric weave form simulation based on the characteristics of yarn, Kansei Engineering International Journal, 11(4), $2012,277-282$

[16] S. Adanur, S. Jaget, Woven fabric design and analysis in 3D virtual reality, Part 1: Computer aided design and modeling of interlaced structures, Journal of the Textile Institute, 104.7, 2013, 715-723

[17] I. Dabolina, A. Vilumsone, The role of the latest clothing CAD/CAM system applications in the educational process, Material Science, 2012, 1691-3132, 7

[18] Y. Liu, D. Zhang, M. Yuen, A survey on CAD methods in 3D garment design, Computers in Industry, 61.6, 2010, 576-593

[19] Y. Zhong, Fast virtual garment dressing on posed human model, Journal of Fiber Bioengineering and Informatics, 1.1, 2008, 21-28

[20] H. Liu, Y. Zhong, S. Wang, Numerical stability of integration methods used in cloth simulation, Journal of Fiber Bioengineering and Informatics, 3.2, 2010, 106-110

[21] X. Zeng, An overview on computational techniques in textile engineering, Journal of Fiber Bioengineering and Informatics, 1.3, 2008, 177-184

[22] M. Takatera, Technical vision of evaluation and computerization for the textile information, Sen I Gakkaishi, 70.9, 2014, 589 (in Japanese)

[23] Z. J. Grosicki, W. Watson, Watson's Textile Design and Color: Elementary Weaves and Figured Fabrics, Newnes-Butterworths, 7th edition, 1975, 150-168

[24] Information at Organization for Small \& Medium Enterprises and Regional Innovation, JAPAN, (http://www.smrj.go.jp/keiei/seni/info/pub/archives/)

[25] K. Sasaki, H. Shimizo, The effects of physical properties of clothes on the tactile sensation by vision, Bulletin of Faculty of Education of Utsunomiya University, 2(58), 2008, 49-58 (in Japanese)

[26] F. T. Peirce, 5-The geometry of cloth structure, Journal of the Textile Institute Transactions, 28(3), 1937, T45-T96 